\title{
Impact of Performance Level and Group Composition on Student Learning during Collaborative Exams
}

\author{
Yingjun Cao and Leo Porter \\ Department of Computer Science and Engineering \\ University of California, San Diego \\ La Jolla, CA, USA
}

\begin{abstract}
Collaborative exams have shown promise for improving student learning in computing. Prior studies have focused on benefits for all students, whereas this study seeks to refine our understanding of which students benefit and how group composition impacts that benefit. Using a crossover experimental design, the study first investigates whether students from differing performance levels (low, medium, or high) benefit from the collaborative exam. We find that students in the middle of the class (neither high nor low performers) tend to benefit strongly from the collaborative exam. Second, we explore whether group composition based on performance levels impacts the performance of members of the group. The results suggest more homogeneous groups (i.e., students in the group are at similar performance levels) are beneficial whereas students in groups with high heterogeneity do not experience significant performance differences between the pre-test and post-test.
\end{abstract}

\section{Categories and Subject Descriptors}

K.3.2 [Computer Science Education]: Computer and Information Science Education

\section{Keywords}

Collaborative Exam; Group Composition; Performance Level; CS1

\section{INTRODUCTION}

Collaborative exams, especially two-stage exams, have shown promising results in improving student grades [10, 15] and promoting student learning $[6,12]$ outside of computing fields. Two-stage exams start with a normal individual exam which is then immediately followed by a group exam where students work in small groups to solve a set of problems $[22,28,29]$. Due to the high-stakes nature of an exam, students are expected to fully participate in the problemsolving process. As a novel hybrid form of summative and

Permission to make digital or hard copies of all or part of this work for personal or classroom use is granted without fee provided that copies are not made or distributed for profit or commercial advantage and that copies bear this notice and the full citation on the first page. Copyrights for components of this work owned by others than ACM must be honored. Abstracting with credit is permitted. To copy otherwise, or republish, to post on servers or to redistribute to lists, requires prior specific permission and/or a fee. Request permissions from permissions@ acm.org.

ITiCSE '17 July 03-05, 2017, Bologna, Italy

(C) 2017 ACM. ISBN 978-1-4503-4704-4/17/07 . \$ $\$ 15.00$

DOI: http://dx.doi.org/10.1145/3059009.3059024 formative assessments, this exam format has been shown to statistically improve short-term student learning in computing through a recent controlled study [4].

Although collaborative exams have been shown to promote student learning in computing, it is unknown how the performance level of a student might impact their potential learning benefit from the exam. Moreover, it is unknown how group composition impacts learning given evidence that group composition can impact team effectiveness [1]. In prior studies on two-stage exams, students usually self-select team members $[12,27]$ or are randomly assigned to groups [4].

In this study, we investigate how collaborative exams affect student learning based on students' performance level, both individually and collaboratively in a group. We use students' normalized pre-test scores on specific topics as the metric for students' performance level. This is the first study in computing, to the best of our knowledge, to examine directly the impact of student performance levels on their learning and on group effectiveness during collaborative exams. To perform this study, we adopted a crossover design $[4,12]$ in order to measure students' performance differences between the pre-test and post-test. The crossover design modifies standard two-stage exams by placing students randomly into control or experimental conditions. All students take a normal individual exam for the first part of a midterm exam (i.e., a pre-test). Students in the control group for a topic then spend test time individually answering questions. Students in the experimental group for the same topic then answer those same questions but do so in small groups. In the end, all students complete a quiz a few weeks later (i.e., a post-test). The value of the crossover design is it enables all students to be in the control group for one topic and the experimental group for another topic (see Section 3 for details). Additionally, the format helps control for time-on-task by having a student spend time in the control group working individually.

Employing this study design, we find that mid-performing students show significant improvement in their post-test compared with their per-test after collaborative exams while low- and high-performing students tend to have insignificant performance differences. Using group members' performance levels in the pre-test to measure group heterogeneity, we find that low and moderate heterogeneity groups statistically benefit from collaborative exams. These results suggest the homogeneous grouping of students by performance when employing group exams. 


\section{BACKGROUND}

Collaborative Exam Effectiveness: Although the primary purpose of formative assessments is to promote student learning, summative exams can also offer students the opportunity to learn. Studies on the "testing effect" [11, 23] have long shown that testing can improve knowledge retention. Tests and exams also promote learning indirectly as students usually only study on topics that they believe will appear in subsequent tests [3]. Given the prevalence of exams and tests in higher education, researchers have begun to examine how to additionally leverage testing as a learning tool [24]. In particular, two-stage exams, as a variant of collaborative exams [25], have been shown to improve student learning outside of the computing field $[6,12]$. In computing, work by $\mathrm{Yu}$ et al. [27] has reported that students have positive attitudes towards this testing format. That study also examined student learning through the use of isomorphic questions on midterms and final exams to measure two-stage exam's effectiveness but did not find significant performance gains. In another recent work in computing, Cao and Porter employed a controlled study and found that the group portion of collaborative exams can provide short-term learning benefits for students overall [4].

Collaborative Exam Impact by Performance Level: Researchers outside of computer science have examined if collaborative exams benefit students with different performance levels. Students are categorized based on their exam or quiz scores before the collaborative test. Then performance difference between pre- and post-tests are further explored for each category of students. In the setting of a statistical methods course, Dahlstrom [7] uses pre-test scores of students to rank them into low (below $50 \%$ in the pretest) and high (above 50\%) categories. Using a pre-post experimental design, the study shows low-performing students have significant performance gains in the post-test, both on repeated questions and new questions. No statistically significant results were observed for high-performing students. In another study in biology by Leight et. al. [17], the authors categorized students using their previous exam scores (A to F) and examined the impact of collaborative exams on knowledge retention. The conclusion was there was no difference in benefit by performance levels. In a study in geology [12], students were categorized into three quantiles based on their overall pre-test scores. The authors found that there is no statistical difference on performance among the three quantiles. To the best of our knowledge, no similar work exists in computing.

Collaborative Exam Impact by Group Heterogeneity: Collaborative exams use group work to promote learning. Research in organizational studies have shown that group performance is closely related to team members' characteristics [5, 16, 20, 26]. In the computing field, software engineering researchers have studied team members' attributes that impact team performance. For example, Faraj et. al. [9] found that communication and expertise are strongly correlated with team performance. Similarly, Gorla et. al. [13] incorporated personality into team productivity measure. While these studies inform this work, the group work during collaborative exams is different from common collaborative teamwork in software engineering in two key aspects: (1) the solutions to exam problems may require less creativity than those in software engineering projects; (2) students in the exam setting are time pressured-having little time to com- plete the task. These unique attributes of group work during collaborative exams may create interaction styles among team members which are different from those in prior studies. As such, this study examines if heterogeneity of performance levels in groups impacts student learning.

\section{METHOD}

Both sections of CS1.5 in Java offered in the Spring of 2016 at a large North American university were selected for this study. The total enrollment was 278 at the beginning of the term. At this institution, CS1.5 is a gateway course into the major and grades in the course impact students' ability to transfer into the computer science major. The lectures for these sections were held back-to-back. The course was structured to have two midterms and three quizzes. The pattern of the these assessments is quiz 1, midterm 1, quiz 2, midterm 2, and quiz 3. Each quiz and midterm are 2week apart with the two-week window being a convenient choice for the flow of the course. Peer instruction [21] is used in this course. The instructor has taught this course twice using peer instruction and is one of the authors of this work.

For purposes of this study, both sections of CS1.5 are treated as one large section throughout the analysis. Using the consent form approved by the institutional review board, 247 students consented to participate in this research project by the time of the first midterm, and 228 of these students were present during the second midterm.

\subsection{Experimental Design}

The cross-over design from Cao et. al. [4] was used to measure potential performance differences with collaborative exam as the experimental condition. Figure 1 provides an overview of this design.

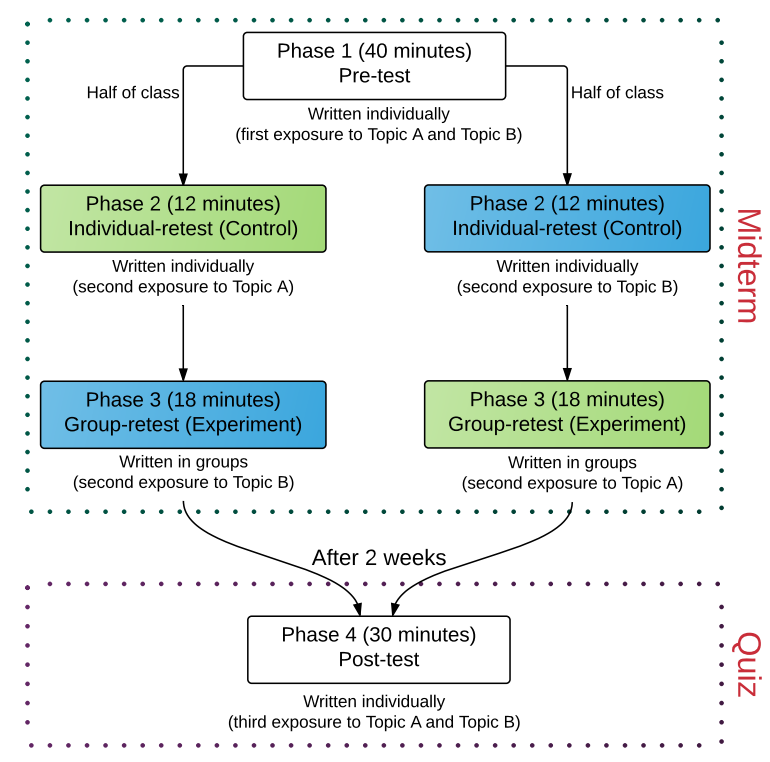

Figure 1: Experimental procedure. Students are randomly assigned to either the left path or the right path. The experimental phase and the control phase have the same questions per topic and students experience these questions either during the individual or group retest. 
Two independent experiments were conducted with midterm 1 and quiz 2 forming an experiment and midterm 2 and quiz 3 forming the second experiment. The phases of the experiment are:

Phase 1 - Pre-test: The first portion of the midterm consists of students completing the individual exam (i.e. pretest). The individual exam includes one problem on Topic A and one on Topic B. Individual exam papers are collected at the end of phase 1 .

Phase 2 - Individual-Retest (control): Each student is given a retest paper with one in-depth problem on either Topic A or Topic B, depending on their path through Figure 1. Students are given 12 minutes to complete this task. The purpose of this phase is to help separate out the impact of spending more time on the topic alone during the retest (control condition) from spending more time on the topic in a collaborative setting described next (experimental condition).

Phase 3 - Group-Retest (experiment): The third phase is the experimental condition where students solve problems on the different topic from that they solved in the second phase (questions, per topic, are identical in the Individualand Group-Retest phases). During this phase, students work together in groups of four to solve the problem with some rare cases of 3-person groups because of the number of student present during the midterm. This phase lasts 18 minutes which is slightly longer than the control phase to allow for students to fully engage in collaborative work. The group-retest is offered at the end of the midterm to avoid having to refocus students from group to individual work and to avoid groups hearing discussion of questions which they might later see during an individual-retest.

Phase 4 - Post-test: The 30-minute quiz, i.e. post-test, is given 2 weeks after the midterm. Similar questions to the pre-test for Topic A and Topic B are given in this phase. However question difficulty (and content) on each topic may vary between assessments.

To summarize, for an experiment, there are two assessment scores per student:

- Individual pre-test: Each student has a score for Topics A and B in the individual test (i.e. phase 1 in Figure 1) of the midterm. These scores also indicate the performance level of a student on these concepts.

- post-test: Again, each student will have a score for Topic A and another score for Topic B in the follow-up quiz (2 weeks after the midterm).

We compare scores between the post-test and pre-test to measure the performance difference for each student per topic. The performance difference $(\mathrm{PD})$ can be thought of as the change in student performance on the topic between the pre- and post-test. For students in each of the control and experimental condition, we calculate this performance difference and examine differences between these groups. Note that because questions were not necessarily of equal difficulty, a negative performance difference does not imply students suffered a loss in understanding. A negative performance difference likely implies the post-test had simply more difficult questions.

The topics ("A" and "B") in each experiment were selected based on the normal flow of the course and the relationships between the topics. For the first experiment, the two topics were method overloading and overriding (Topic A) and constructors (Topic B). Both topics are closely related to inheritance in Java. For the second experiment, the two topics were polymorphism (Topic A) and Recursion (Topic B). These two topics are not considered to be closely related. All exams and quizzes are closed book and closed notes. Due to space constraints, we omit sample questions here, and ask interested readers to examine sample questions from our prior work (Cao and Porter [4]).

\subsection{Research Questions}

This work explores two related research questions on the impact of collaborative exams on student learning categorized by students' performance level, both individually and collaboratively.

RQ1: How does the performance level of a student impact the learning benefit from collaborative exams?

RQ2: Does group composition affect student learning during collaborative exams?

\subsection{Measurement Metric}

To evaluate the effectiveness of the group portion of a two-stage exam, we compare performance differences of students exposed to the control or experimental condition. Between the pre-test and post-test, the only in-class difference among students' exposure to each topic is either via the individual-retest (control) or the group-retest (experiment). Every student experiences two performance differences from each experiment: one from the individual-retest, and one from the group-retest. Without losing generality, assume a student is exposed to Topic A in the individual retest and Topic B in the group retest (i.e., the left branch in Figure 1). Suppose the student's scores for Topics A and B in the pre-test are $\operatorname{Pre}_{A}$ and $\operatorname{Pre}_{B}$. Similarly, the scores of the student in the post-test on the two topics are Post $_{A}$ and Post $_{B}$. The performance difference of this student from individual retest is $P D_{\text {individual }}=\operatorname{Post}_{A}-\operatorname{Pre}_{A}$, and that from group retest is $P D_{\text {group }}=$ Post $_{B}-$ Pre $_{B}$. We then calculate $P D_{\text {diff }}=P D_{\text {group }}-P D_{\text {individual }}$ which is the measurement of the relative effectiveness of collaborative exams compared to simply having extra time on the topics.

The metric above is the absolute performance difference. An alternative metric, normalized learning gain (or normalized performance difference here), was also explored [19]. However, we observed that this variant tends to exaggerate outcomes on easier topics/questions and because the overall conclusions were the same using normalized performance difference, we focus on absolute performance difference in presenting the results.

\subsubsection{Student Performance Level}

To measure the performance level of a student in each experiment, we used the average of z-scores on Topics A and B. The normalization process removes scaling effect (different difficulties and/or distributions) from two different problems before combining them together. This metric for performance level is different from previous studies [12, 18] which measured students' performance level with their overall pre-test scores. We believe that even though a student's scores on pre-selected topics are correlated with their overall pre-test scores (Pearson $r=.408, p<.001$ in our study), our proposed metric for performance measure may offer a more accurate measurement of performance level by focusing solely on the topics in question. 
To rank students into commonly accepted categories of low, mid, and high performance level, we coded students who are within one standard deviation (SD) from the mean as mid-performing. Those who are below one SD from the mean is ranked as low-performing and those who are above one SD from the mean as high-performing. This strategy resulted in $22.3 \%$ of students binned to the low category (106 students in total), $65.5 \%$ to the mid category (311 students in total), and $12.2 \%$ to the high category (58 students in total). This ranking resulted in imbalanced groups of low, mid, and high performing students. However, simply binning by thirds produced questionable cutoffs whereas our categorization method yields more meaningful separation between student groups.

\subsubsection{Group Categorization}

Group heterogeneity is measured using the standard deviation of group members' performance. The larger the standard deviation, the more heterogeneous the group is with regard to group members' performance level. Each group's standard deviation is used to classify each group into three equal quantiles - low, mid, or high heterogeneity.

All categorization and rankings are performed within each experiment first. Then the results from both experiments are pooled together for analysis. For comparisons of PD, statistical significance is set at the $p \leq 0.05$ level.

\section{RESULTS}

\subsection{Experimental Results}

For each experiment, we cross-tabulated student exam data with experimental conditions and pre-selected topics (individual-retest as control and group-retest as experimental). The differential PD is obtained for each student per experiment. Table 1 provides these results for the class overall.

\begin{tabular}{|c|c|c|c|c|c|c|c|c|}
\hline \multirow{2}{*}{\multicolumn{2}{|c|}{ Topic }} & \multicolumn{2}{|c|}{ Pre-test } & \multicolumn{2}{|c|}{ Post-test } & \multirow{3}{*}{$\begin{array}{l}\text { Ind. } \\
\text { PD }\end{array}$} & \multirow{2}{*}{$\begin{array}{c}\text { Group } \\
\text { PD }\end{array}$} & \multirow{2}{*}{$\begin{array}{l}\text { Diff. } \\
\text { PD }\end{array}$} \\
\hline & & Ctrl & Exp & Ctrl & Exp & & & \\
\hline \multirow{2}{*}{ Exp1 } & $\mathbf{A}$ & 55.0 & 52.4 & 67.5 & 76.3 & & 23.9 & \multirow{2}{*}{10.9} \\
\hline & B & 71.1 & 69.8 & 70.3 & 77.4 & -0.9 & 7.6 & \\
\hline \multirow{2}{*}{ Exp2 } & $\mathbf{A}$ & 85.8 & 83.3 & 67.0 & 68.6 & -18.8 & -14.6 & \multirow[b]{2}{*}{2.0} \\
\hline & B & 89.1 & 87.8 & 79.7 & 77.2 & -9.5 & -10.6 & \\
\hline
\end{tabular}

Table 1: Raw data from controlled experiments.

These results are provided for two reasons. The first is as a reminder that because questions on the Pre-test and Posttest may vary in difficulty, we may find students achieving lower scores on the post-test than the pre-test. The second reason is to show how these scores by topic and by experimental condition are combined into an individual PD and group PD. Concept B in the second experiment (recursion) shows that students in the experimental condition did worse than that from the control group. However, this study is focused on the benefit from the group exam over the individual retest, which is provided as the differential in group and individual PD. For both experiments, the differential PD was positive, showing a measurable benefit from the group exam. We pool data from both experiments after normalization for each experiment to remove potential scaling effects. For the remainder of the study, we focus on the differential PDs on the pooled dataset.

\subsection{RQ1: How does the performance level of a student impact the learning benefit from collaborative exams?}

For each student, the average performance level on the two pre-selected topics in the pre-test per experiment is calculated as discussed in Section 3.3.1. Student results are then binned based on their performance level into low, mid, or high performing students. Figure 2 provides the PD for students based on performance level. One-sample $t$-tests were used to calculate significance for differential PD. There are no statistically significant differences for both low-performing and high-performing students. However, for mid-performing students, collaborative exams statistically improve student learning with a small effect size (Cohen's $d$ ).

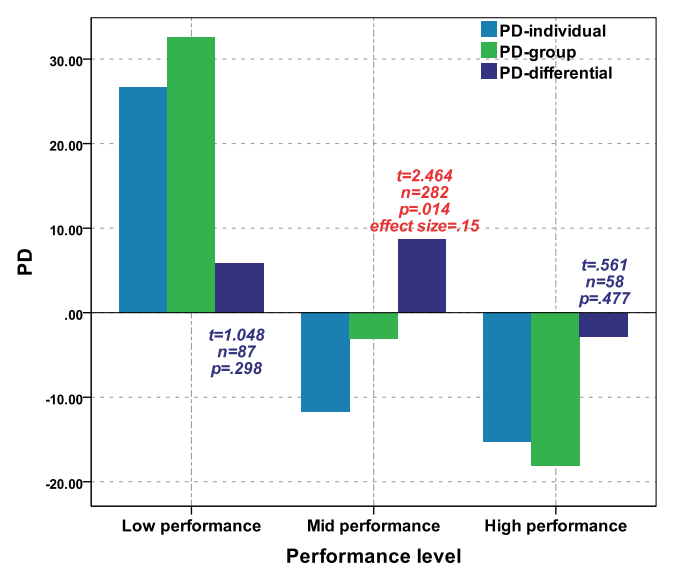

Figure 2: Student PD categorized by student performance level. Statistics for differential PD are provided; red font indicates statistical significance.

\subsection{RQ2: Does group composition affect stu- dent learning during collaborative exams?}

We next examine the benefit from the group exam based on the heterogeneity of performance levels among group members. The differential PD (PD of the experimental minus that of the control condition) for each binning of heterogeneity appears in Figure 3. One-sample $t$-tests are used to examine if differential PDs are zero. These results show

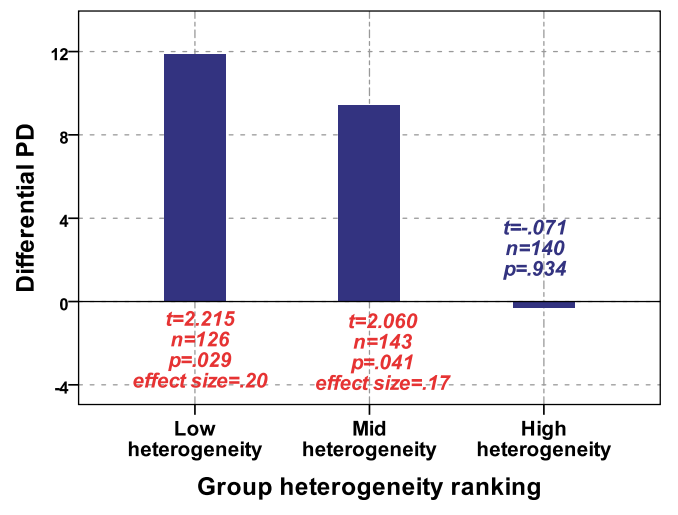

Figure 3: Differential PD categorized by group heterogeneity. Statistics for each category are provided; red fonts indicate statistical significance. 
that groups with both low- and mid-levels of heterogeneity experience statistically significantly benefit from the group portion of the exam. High-heterogeneity groups do not.

The group composition within highly heterogeneous groups requires there be both high- and low-performing students. Homogeneous groups may consist of students from within any level of performance. Moreover, recall that the majority of students were classified as mid-performance, so a group of all low-performing students is less likely than one of all mid-performing. Figure 4 provides the number of students in each type of group based on their performance level.

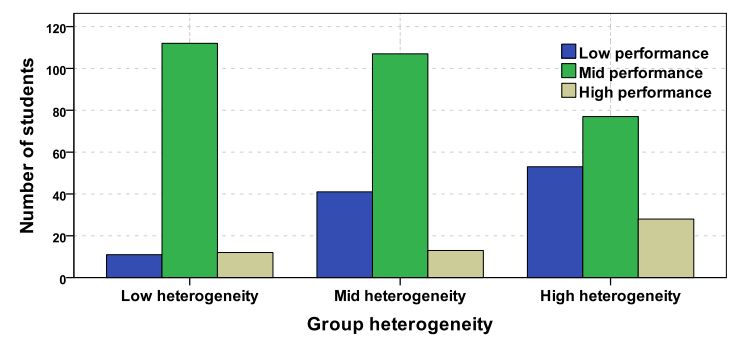

Figure 4: Composition of groups based on heterogeneity and student performance level.

These results show that high-heterogeneity groups have more low- and high-performing students than those with low heterogeneity. We also further stratified students for each heterogeneity group based on their performance level. It produces nine categories such as students with low-performance level in a low-heterogeneity group, students with mid-performance level in a high-heterogeneity group, etc. However, when dividing students into nine categories, the sample sizes in these grouping were small enough to limit our ability to draw reasonable conclusions or to perform meaningful statistical analysis. Also, no consistent pattern was observed from these sub-groupings.

\section{DISCUSSION}

RQ1 - Performance Level: For low-performing students, they experience positive learning benefit from both the individual retest and group exam. As a result, the additional benefit of the group exam lacks statistical significance. This may indicate that low-performers learn more simply by spending more time on the subject, either individually or collaboratively, or that they spend more time after the exam preparing for the next quiz.

The category which appears to benefit the most from collaborative exams is mid-performing students. Figure 2 showed that this group experienced negative PDs for both the control and experimental condition, likely due to differences in difficulty. Those students in the experimental condition experienced a much smaller drop in performance than those in the control condition. This difference, along with the larger sample size, causes the differential PD to be significant. These results confirm those from existing studies that collaborative exams tend to improve knowledge retention [6, 8]. For high-performing students, they have a similar amount of negative PDs from both the groupretest and individual-retest. Therefore, differential PD was minimum. This result partially confirms the conclusion by Dahlstrom [7] that high-performing students do not show significant improvements from collaborative tests.
RQ2 - Group Heterogeneity: The observation that lowand mid-level group heterogeneity tend to produce high levels of student PDs is quite intriguing as existing research in educational psychology indicates that a more diverse group tends to be more creative in solving problems [20]. We believe that the aforementioned unique characteristics of exams may be the cause. Students need to complete a set of challenging questions during a short period. We suspect this may limit the role of group creativity during brief, highstakes summative exams [2].

In homogeneous groups, the smaller divide in performance between students may allow for easier communication in general which leads to the improved group learning. Specifically, we suspect homogeneous groups have more student engagement as the students may feel more comfortable interacting with peers with similar levels of understanding. We also believe that it takes less time for the students to explain their thinking to similarly knowledgeable students. In addition, homogeneous groups may also tend to reach a solution that is agreeable to most team members while a highly heterogeneous group's solution may simply be provided by the high-performing member of the group.

Figure 4 also shows that high heterogeneity groups tend to have more low- and high-performing students. As these students tend to have small PDs as shown in RQ1, it could also explain why high heterogeneity groups tend to have limited PDs. It is difficult to distinguish if the lower PDs for low- and high-performing students is the cause, or the effect, from the degree of group heterogeneity.

Overall, our results seem to indicate that homogeneous grouping strategies should be explored to maximize the impact of collaborative exams.

\section{Threats to Validity}

Defining Student Performance Level: Student performance level was ranked based on standard-deviation on scaled pre-test results. We selected this method as we believed it classified students more accurately as within one performance group than another. However, by using standard deviation, we invariably had a larger sample size for our mid-performers and smaller sample sizes for our low- and high-performers. The sample size differences may impact our results and certainly impact the $p$ value for statistical tests. However, we attempted other ranking approaches, such as equal quantiles, for determining students' performance level and the same overall conclusions were found.

Lack of Isomorphic Questions: The questions given during the pre- and post-tests were similar but were not identical. We thoroughly examined the questions from the pretests and post-tests and believe they are quite similar in terms of content and associated concepts. However, because the questions were different the questions could unintentionally test different concepts or skills between the pre-test versus the post-test. Future work could examine using isomorphic questions to reduce this confound.

\section{CONCLUSION}

This study shows that the level of student performance impacts the benefit they receive from collaborative exams in computing. Mid-performing students are found to experience significant learning benefit from the collaborative exams whereas low- and high-performing students do not. Group heterogeneity, based on the difference between per- 
formance level of team members, also impacts the benefit offered from collaborative exams. Groups with low levels of heterogeneity experience significant learning benefit for students. These results suggest that student groups in collaborative exams should be formed to be homogeneous regarding student performance. Although there has been similar observations on group dynamics in pair programming [14], this result is contrary to results in other domains where more heterogeneous groupings are beneficial. Therefore, we recommend further study on this topic.

\section{ACKNOWLEDGMENTS}

This work was partially supported by NSF DEERS project $(1525028,1525173,1525373)$. The authors want to thank Daniel Zingaro for his feedback on an early draft of this work, and also want to thank the anonymous reviewers for their helpful suggestions.

\section{REFERENCES}

[1] M. R. Barrick, G. L. Stewart, M. J. Neubert, and M. K. Mount. Relating member ability and personality to work-team processes and team effectiveness. Journal of Applied Psychology, 83(3):377-391, 1998.

[2] D. Baud. Reframing assessment as if learning were important. In Rethinking assessment in higher education: learning for the longer term., pages 14-25. Oxford: Routledge, 2007.

[3] J. Biggs. Enhancing teaching through constructive alignment. Higher Education, 32(3):347-364, 1996.

[4] Y. Cao and L. Porter. Evaluating student learning from collaborative group tests in introductory computing. In SIGCSE, pages 99-104, 2017.

[5] M. A. Carpenter. The implications of strategy and social context for the relationship between top management team heterogeneity and firm performance. Strategic Management Journal, 23(3):275-284, 2002.

[6] R. N. Cortright, H. L. Collins, D. W. Rodenbaugh, and S. E. DiCarlo. Student retention of course content is improved by collaborative-group testing. Advances in Physiology Education, 27(3):102-108, 2003.

[7] Ö. Dahlström. Learning during a collaborative final exam. Educational Research and Evaluation, 18(4):321-332, 2012.

[8] M. L. Epstein, A. D. Lazarus, T. B. Calvano, K. A. Matthews, R. A. Hendel, B. B. Epstein, and G. M. Brosvic. Immediate feedback assessment technique promotes learning and corrects inaccurate first responses. The Psychological Record, 52:187-201, 2002.

[9] S. Faraj and L. Sproull. Coordinating expertise in software development teams. Management Science, 46(12):1554-1568, 2000.

[10] M. Fengler and P. M. Ostafichuk. Successes with two-stage exams in mechanical engineering. CEEA, pages 1-5, 2015.

[11] A. I. Gates. Recitation as a factor in memorizing. Number 40. Science Press, 1922.

[12] B. H. Gilley and B. Clarkston. Collaborative testing: Evidence of learning in a controlled in-class study of undergraduate students. Journal of College Science Teaching, 43(3):83-91, 2014.
[13] N. Gorla and Y. W. Lam. Who should work with whom? building effective software project teams. Communications of the ACM, 47(6):79-82, 2004.

[14] N. Katira, L. Williams, E. Wiebe, C. Miller, S. Balik, and E. Gehringer. On understanding compatibility of student pair programmers. In SIGCSE, pages 7-11, 2004 .

[15] K. Knierim, H. Turner, and R. K. Davis. Two-stage exams improve student learning in an introductory geology course: Logistics, attendance, and grades. Journal of Geoscience Education, 63:157-164, 2015.

[16] R. A. Layton, M. L. Loughry, M. W. Ohland, and G. D. Ricco. Design and validation of a web-based system for assigning members to teams using instructor-specified criteria. Advances in Engineering Education, 2(1):1-28, 2010.

[17] H. Leight, C. Saunders, R. Calkins, and M. Withers. Collaborative testing improves performance but not content retention in a large-enrollment introductory biology class. CBE Life Sciences Education, 11(4):392-401, 2012.

[18] G. L. Macpherson, Y. J. Lee, and D. Steeples. Group-examination improves learning for low-achieving students. Journal of Geoscience Education, 59(1):41, 2011.

[19] J. D. Marx and K. Cummings. Normalized change. American Journal of Physics, 75(2007):87, 2007.

[20] B. A. Nijstad and C. K. W. De Dreu. Creativity and group innovation. Applied Psychology, 51(3):400-405, 2002.

[21] L. Porter, C. Bailey Lee, B. Simon, and D. Zingaro. Peer instruction: do students really learn from peer discussion in computing? ICER, pages 45-52, 2011.

[22] B. G. W. Rieger and C. E. Heiner. Examinations that support collaborative learning: The students' perspective. Journal of College Science Teaching, 43(4):41-48, 2014.

[23] H. L. Roediger and J. D. Karpicke. The power of testing memory basic research and implications for educational practice. Perspectives on Psychological Science, 1(3):181-210, 2006.

[24] H. L. Roediger III, P. K. Agarwal, M. A. McDaniel, and K. B. McDermott. Test-enhanced learning in the classroom: long-term improvements from quizzing. Journal of Experimental Psychology: Applied, 17(4):382, 2011.

[25] S. A. Stearns. Collaborative exams as learning tools. College Teaching, 44(3):111-112, 1996.

[26] M. A. West. Sparkling fountains or stagnant ponds: An integrative model of creativity and innovation implementation in work groups. Applied Psychology: An International Review, 51(3):355-424, 2002.

[27] B. Yu, G. Tsiknis, and M. Allen. Turning exams into a learning experience. In SIGCSE, pages 336-340, 2010.

[28] R. F. Yuretich, S. a. Khan, R. M. Leckie, and J. J. Clement. Active-learning methods to improve student performance and scientific interest in a large introductory oceanography course. Journal of Geoscience Education, 49:111-119, 2001.

[29] J. F. Zipp. Learning by exams : The impact of two-stage cooperative tests. Teaching Sociology, 35(1):62-76, 2007. 